\title{
Non-academic factors influencing the development of empathy in undergraduate nursing students: a cross-sectional study
}

Nancy Berduzco-Torres ${ }^{1 \dagger}$, Pamela Medina ${ }^{1 \dagger}$, Montserrat San-Martín², Roberto C. Delgado Bolton ${ }^{3,4,5}$ and Luis Vivanco ${ }^{4,5^{*}}$

\begin{abstract}
Background: Empathy is described as a core competence of nursing. There is abundant research evidence supporting that empathy varies according to personal characteristics and targeted training. The aim of this study was to characterize non-academic factors (personal and environmental) influencing the development of empathy in undergraduate nursing studies who are not receiving a targeted training in empathetic abilities in their nursing schools.

Methods: A cross-sectional study was performed in the three nursing schools located in Cusco city, Peru (two private and one public). The Jefferson Scales of Empathy, Attitudes toward Physician-Nurse Collaboration, and Lifelong Learning, the Emotional Loneliness Scale for Adults, and the Scale of Life Satisfaction, were applied as the main measures. Also, information regarding gender, nursing school, and age, were collected. After psychometric properties were assessed, all measures were used in the development of a multivariate regression model to characterize factors of influence in empathy.
\end{abstract}

Results: In a sample composed by 700 undergraduate nursing students ( 72 males and 628 females), a multivariate linear regression model was created. This model explained the $53 \%$ of variance of empathy and fitted all conditions necessary for inference estimations. Teamwork abilities, loneliness, age, sex, subjective well-being, and nursing school, appeared as factors influencing the development of empathy in patients' care.

Conclusions: Findings have indicated that, in absence of a targeted training, individual characteristics and characteristics associated with social and family environments play an important role of influence in the development of empathy in nursing students. These findings are also in consonance with others previously reported in different cultural settings including high-, middle- and low-income countries.

Keywords: Nursing students, Empathy, Loneliness, Personality development, Sex characteristics, Universities

\section{Introduction}

Empathy has been described as an important component of professionalism in healthcare and especially in those

\footnotetext{
* Correspondence: Ivivanco@riojasalud.es

${ }^{4}$ Centro Nacional de Documentación en Bioética, C/Piqueras 98, 26006 Logroño, Spain

${ }^{5}$ Centro de Investigación Biomédica de La Rioja (CIBIR), C/ Piqueras 98, La Rioja 26006 Logroño, Spain

Full list of author information is available at the end of the article
}

disciplines that are in direct contact with patients, such as nursing [1]. In fact, it is widely accepted that the ability of nurses to empathize with their patients is a desirable quality, and that patients want empathic and emotionally competent nurses. Moreover, there is evidence that empathy enables nurses to handle difficulties better [2]. According to some authors [3], nurse educators play an important role of responsibility as providers

(c) The Author(s). 2021 Open Access This article is licensed under a Creative Commons Attribution 4.0 International License, which permits use, sharing, adaptation, distribution and reproduction in any medium or format, as long as you give appropriate credit to the original author(s) and the source, provide a link to the Creative Commons licence, and indicate if changes were made. The images or other third party material in this article are included in the article's Creative Commons licence, unless indicated otherwise in a credit line to the material. If material is not included in the article's Creative Commons licence and your intended use is not permitted by statutory regulation or exceeds the permitted use, you will need to obtain permission directly from the copyright holder. To view a copy of this licence, visit http://creativecommons.org/licenses/by/4.0/ The Creative Commons Public Domain Dedication waiver (http://creativecommons.org/publicdomain/zero/1.0/) applies to the data made available in this article, unless otherwise stated in a credit line to the data. 
of an education that engenders empathic understanding. In consonance with this, Richardson and colleagues have suggested the inclusion of targeted training in academic programs, such as "nursing therapeutics" methodology, teaching nursing students how to care using compassion and empathy [4]. In recent years, similar training experiences based on communication and understanding skills have demonstrated a positive effect in the enhancement of empathy in medical $[5,6]$ and nursing students $[7,8]$.

However, there are still many countries in which targeted training focused on acquiring and improving empathic abilities is a pending task $[5,6,9]$. In the specific case of Peruvian medical and nursing schools, targeted training programs focused on the acquisition of empathic abilities are included in their curricula in a few number of cases [6]. In the majority of cases, this type of knowledge is expected to be acquired as part of the clinical training that medical and nursing students receive in advanced stages of their studies. Recent studies have highlighted that the lack of appropriate support, role modelling, and focused training in Peruvian institutions is associated not only with no improvement in empathy, but also with a deterioration of other aspects such as ethics and emotional wellbeing $[9,10]$. Under these circumstances, it is clear that personal skills and nonacademic environments (such as family and cultural ones) have acquired more relevance as possible main sources of influence in the development of empathic abilities in nursing students, especially when targeted training activities on empathetic abilities are missing or limited.

\section{Background}

When referring to clinical interactions, empathy has been described as a professional competence that is principally cognitive, more than affective or emotional. Such distinction between cognition and emotion (and correspondingly, between empathy and sympathy) may not seem as important in situations different from clinical work, in which both elements could have a similar importance [11]. However, in clinical encounters, establishing a relationship with the patients mainly based on a cognitive or an emotional response can derive in different outcomes for patients and for healthcare professionals [12]. In clinical encounters, cognitive mental processing primarily refers to an advanced intellectual process that often involves social perception, analysis of information, and generation of appropriate responses based on the ability to understand patients' concerns, which are some of the main components of clinical empathy. In contrast, an emotional response consists primarily in a more primitive mental process, where the response is consequence of an affective resonance in healthcare professionals of the emotions observed in their patients, which is a characteristic element of a sympathetic reaction. According to Hojat [13], "the distinction between sympathy (also known as emotional empathy, or vicarious empathy) and empathy (also known as cognitive empathy, or clinical empathy in the context of patient care) has important implications for the clinician-patient relationship because joining the patient' emotions, a key feature of sympathy, can impede clinical outcomes". In this sense, it could be recommended for a healthcare professional to sense the patient's feelings only if it does not impede his/her professional work [14]. Otherwise, it can be difficult to keep a sense of whose feelings belong to whom [15]. Fernandez and Zahavi recently stated in this matter, "Empathy in its most basic sense offers a direct and immediate form of other-understanding-one that doesn't require us to reproduce or share the other's experience" [12]. Without concluding that emotions are, per se, a barrier in the establishment of an empathic relationship with patients, their role is subordinated under the control of cognitive components. Studies on socialneuroscience recently have shown evidence demonstrating that an affective sharing may act as a gain antecedent to empathic understanding, while cognitive components are important for representing the mental states of self and other, necessary to make decisions in a clinical context [16].

On this basis, there are three elements that are generally accepted as components of empathy in clinical contexts [13]: (i) comprehending patients' experiences, concerns and perspectives; (ii) a good and clear communication; and (iii) an intention to help, expressed in a compassionate (benevolent) attitude aimed at taking care of a sick person. The first two components, mostly described as "mind's eye" (understanding) and "third ear" (listening), have been associated with socio-emotional processes in experimental studies, such as social knowledge, social perception, and decision-making [17, 18]. These two components are constitutive of the "empathic curiosity" that, according to Jodi Halpern, encourage an empathic response in clinicians focusing on learning more about what their patients are experiencing and how it affects their attitude towards their treatment [11, 19]. The third component of an empathic response, "intention to help", has been associated with the capability of controlling personal anxiety derived from the exposition to patients' suffering [20]. Neuroimaging studies suggest that in the background of this emotional regulation there is a neural control of brain regions involved in emotional responses, such as the insula, the anterior cingulate cortex, and the periaqueductal gray [16, 21, 22]. When focusing on nurses, an intention to help as the main personal interest has been described as an attribute that allows them to perceive the patient as like self while 
keeping a clear separation between self and the patient [13]. In accordance with this, much of the nursing literature suggests that nurses should not rely on their innate capacity for emotional empathy; instead, they should develop intellectual techniques for understanding their patients in an objective manner that provides distance from their patients' emotional distress [12]. From a phenomenological perspective, some authors complete this approach with the consideration that nurses, based in the altruistic nature of nursing, have more resources to be open to and appreciate the individuality of each patient in their daily work [23].

Regarding possible individual factors influencing the development of empathy in healthcare professions, findings from a large number of studies suggest that women are often more empathic than men, obtaining higher scores in empathy measures [24-27]. These findings are in consonance with others performed in general population where women have shown better indicators in neurological measures related to empathy [28]. Certain genetic predisposition, evolutionary underpinnings, and interpersonal styles, but also social interactions have been described as possible explanations for such gender differences in empathic responses [29]. In medical students, personality [30], personal motivation for studying medicine [31], and career interest [24] have also been described as other influencing factors in the development of empathy.

In relation with the role that the environment plays in the development of empathy, an increasing number of studies have reported evidence supporting its influence at family, social, and cultural levels. In the general population, individuals from communities with greater prosocial indicators, such as higher well-being and higher volunteering rates, have shown higher scores in empathy measures in comparison with those who were living in communities where violence and crime rates were higher [32]. In consonance with this, cross-cultural studies performed with healthcare professionals [33] indicate that culture plays a role of influence in the empathic response to the patients. Studies with medical and nursing students [34-36] also suggest similar findings associated with certain social and cultural environments. Furthermore, studies performed in the United States [37] and recently in Peru [38] have reported that parents and family environments play a role of influence in the development of empathy in medical students.

Taking this into account, this study was designed with the purpose of testing the following hypothesis: In the absence of a targeted training in empathy, its development in nursing students is influenced by individual characteristics and by the influence of the social and family environments. Three objectives were established with this purpose: (i) to identify differences in empathy according to sex and nursing schools groups; (ii) to analyse the type of association existing between empathy and other two professional competences: interprofessional collaboration and lifelong learning abilities; and between empathy measures and students' wellness self-perception (subjective well-being), students' perception of their parents (family environment), academic achievement, and perception of loneliness; and (iii) in those cases in which differences were confirmed, to characterize factors influencing empathy's measurements.

\section{Methods}

\section{Design and participants}

In the second semester of 2019, a cross-sectional study was carried out in the three nursing schools, two private and one public, located in Cusco city in Peru. None of these institutions offered in their curricula a specific course or training program (neither mandatory or elective) focused on empathy or communication and understanding abilities in patient care.

The entire population of undergraduate students enrolled in these institutions was 1030 students. However, for the purpose of this study only undergraduate students attending academic activities in Cusco city were included. Those who were attending academic activities elsewhere when this study was performed, such as communitarian work in isolated rural communities, exchange and internship programs in other institutions, were excluded. Students' participation was voluntary and anonymous. On this basis, the estimation of the minimum sample size required has been calculated with the G*power software, version 3.1.9.7. For this calculation, it was expected to create a regression model based on a linear multiple regression analysis with an effect size between small and medium (Cohen $\left.-f^{2}=0.085\right)$, an alpha equivalent to 0.05 , a power of 0.95 , and at least 5 tested predictors from 10 variables analysed. It was also assumed a $25 \%$ of missing questionnaires (questionnaires that were partially answered). According to this analysis, the minimum sample size required was 319 participants.

\section{Measures}

For measuring empathy, the Healthcare student's version of the Jefferson Scale of Empathy (JSE-HPS), was used. The JSE-HPS (20 items) is answered in a Likert scale from 1 (strongly disagree) to 7 (strongly agree). The JSEHPS follows the same structure of the medical student's version of the JSE (JSE-S). The main difference between both versions is the rewording of terms "medicine" or "physician" to make it clearer for students from healthcare areas different than medicine [13].

To measure inter-professional collaboration (teamwork) between nursing and medicine, the Jefferson Scale 
of Attitudes toward Physician-Nurse Collaboration (JSAPNC) was used [39]. The JSAPNC responds to the definition of teamwork as an ability of nurses and physicians to work together cooperatively, sharing responsibilities for solving problems and making decisions to formulate and carry out plans for patient care. The JSAPNC (15 items) is answered using a Likert scale from 1 (strongly disagree) to 4 (strongly agree).

The Healthcare student's version of the Jefferson Scale of Physician Lifelong Learning (JeffSPLL-HPS) was used to measure attitudes towards lifelong learning [40]. The JeffSPLL measures the development of skills related to information gathering, the use of learning opportunities, and self-motivation [41]. The JeffSPLL (14 items) is answered using a Likert scale from 1 (strongly disagree) to 4 (strongly agree). Similarly to the JSE-HPS, items of the JeffSPLL-HPS were the term "medicine" is used are reworded.

To measure loneliness, the Social and Emotional Loneliness Scale for Adults (SELSA-S), was used. The SELSA-S measures loneliness based in "family", "romantic", and "social" dimensions [42]. The SELSA-S (15 items) is answered in a Likert scale from 1 (strongly disagree) to 7 (strongly agree). Higher scores indicate a greater perception of loneliness. The SELSA-S has shown a high reliability in nurses [2, 43] and in nursing students [34, 44] in Spain and Latin America.

The subjective well-being refers to the emotional and cognitive self-perception of personal life. A Spanish version of the Satisfaction with Life Scale (SWLS) [45] was used as measure of subjective well-being $[46,47]$. The scoring of this version is slightly different to the original one developed in English language by Diener et al. [46]. Each item of the Spanish version is scored with a 5point Likert scale ranging from 1 (strongly disagree) to 5 (strongly agree), while in the English version each item is scored with a 7-point Likert scale. The Spanish version used in this study has been tested in general population [45] and in healthcare professionals [2, 9] showing a high reliability. A high score in the SWLS is associated with high subjective well-being.

The above psychometric instruments mentioned were validated and tested in previous studies with medical and nursing Peruvian students showing excellent psychometric properties and high reliability $[6,9,10]$.

Information regarding age, sex, semester of enrolment, nursing school (public or private), and relationship with parents, were collected through a complementary form. For students' perception of their relationship with their parents, two separate items were used (one for the mother and one for the father). In each case, respondents were invited to answer to the following statement "the relationship with my (mother/father) is" using a Likert scale from 1 (there is no relationship) to 8 (excellent).

\section{Procedures}

Questionnaires together with an information letter were distributed in enclosed envelopes to undergraduate students enrolled in the three nursing schools of Cusco. In order to reduce potential bias in students' responses, the administration of questionnaires were in charge of independent researchers. Students were also informed about the independent nature of this study. Once questionnaires were completed, the participants returned their questionnaires in sealed envelopes following a protocol approved by an independent ethics committee, the "Comité Ético de Investigación de La Rioja" (Ref. CEIC LAR-PI-199). There was no potential risk for participants, and anonymity was guaranteed throughout the entire process.

\section{Statistical analysis}

Only the questionnaires with fully completed items were included into the analysis. The reliability was calculated using Cronbach's alpha coefficient. Values higher than 0.7 were considered satisfactory [48].

As none of the main measures used followed a normal distribution non-parametric tests were performed. For sex (male and female) and nursing school (public and private) variables, a variance analysis (two-way ANOVA) was performed. An interaction effect was also analysed to determine if there were differences in empathy measures defined by a combination of "sex by school". Finally, effect size was calculated using eta-squared value in order to measure the ratio of variance explained in the dependent variable (empathy) by each of the predictors studied while the others were controlled.

With the purpose of determining possible associations between empathy and the following measures: teamwork and lifelong learning abilities, semester of enrolment, loneliness, subjective well-being, and age, a correlation analysis using Spearman's coefficient was performed.

Finally, a multilinear regression analysis using variables with significant differences in previous tests was performed. Those variables were tested as predictors of empathy in order to create a model of inference that can explain empathy's variance. A valid regression model was accepted once the following statistical assumptions were observed: multivariate normality, mean of residuals equal to zero, homogeneity of residuals variance (homoscedasticity), no auto-correlation among residuals, no multicollinearity, and linearity of data. In order to quantify the degree of practical significance of the findings observed in the model obtained, the effect size (Cohen's $f^{2}$ ) was calculated. An effect size equal to 0.02 was interpreted as small, equal to 0.15 was interpreted as medium, and equal to 0.35 was interpreted as large [49].

All analyses were performed using $\mathrm{R}$ statistical software, version 3.6.2 for Windows. The statistical analyses 
of the data also included $l s r$ [50], multilevel [51], apaTables [52], and nortest [53] packages.

\section{Results}

The entire sample included 700 students, corresponding to $68 \%$ of the entire population of undergraduate students for the three nursing schools of Cusco city. From this sample, $72(10 \%)$ were men and 628 (90\%) women. According to schools, 223 (32\%) students were enrolled in the public nursing school, while the others $474(68 \%)$ were enrolled in the two private nursing schools. The mean age was $23(\mathrm{Mdn}=22)$ with a range from 18 to 57 years old $(S D=6$; IQR=7). According to academic achievement (measured by semester enrolled), the range of semesters covered in the entire sample corresponded with the complete undergraduate program of nursing (10 semesters) that is offered in Peruvian nursing schools. All instruments used showed adequate psychometric properties measured by Cronbach coefficients higher than 0.70 in all cases (see Table 1).

Regarding the first objective, results of the two-way ANOVA showed differences in empathy global scores according to "sex" and "nursing school" variables, and also in the interaction of "sex by nursing school". Female students $(\mathrm{M}=102.80 ; \mathrm{Mdn}=104 ; S D=17.74)$ and students enrolled in the public school $(M=107.10$; $M d n=111 ; S D=19.45)$ reported higher scores on empathy in comparison with male students $(M=95.17$; Mdn=97; $S D=17.47)$ and with students enrolled in private nursing schools $(\mathrm{M}=99.56 ; \mathrm{Mdn}=99.50 ; S D=$ 16.55), respectively. Furthermore, the comparison of the interaction between sex and university showed female and male students enrolled in the public school presented the highest and the lowest empathy scores, respectively. The size effect of these three variables in the variance of empathy was small in the case of "sex" $\left(\eta_{\mathrm{p}}{ }^{2}=0.01\right)$, and between small and medium in the case of "nursing school" $\left(\eta_{\mathrm{p}}{ }^{2}=0.04\right)$ and in the interaction of "sex by nursing school" $\left(\eta_{p}{ }^{2}=0.02\right)$ variables (see Table 2).

With regard to the second objective, correlation analyses were performed showing a positive relation between empathy and the following variables: teamwork $(\rho=+0.59 ; p<0.001)$, lifelong learning $(\rho=+0.39$; $p<0.001$ ), and having a positive relationship with the mother $(\rho=+0.08 ; p=0.03)$. On the contrary, a negative association was observed between empathy and loneliness $(\rho=-0.41 ; p<0.001)$, and empathy and age $(\rho=-$ $0.15 ; p<0.001)$. Neither academic achievement $(\rho=+$ $0.06 ; p=0.14)$, having a positive relation with the father $(\rho=+0.06 ; p=0.13)$, or subjective well-being $(\rho=+0.01 ; p=0.72)$ showed a significant association with empathy's global measures, as is shown in Table 3.

Based in the above-observed outcomes, a multiple linear regression analysis was carried out in the entire sample (third objective). This analysis produced a model that explained $53 \%$ of the variability of the JSE measurement $\left(\mathrm{R}^{2}\right.$-adjusted $\left.=0.53 ; \quad F_{(6,652)}=122.50 ; p<0.001\right)$, with a very large effect size $\left(\right.$ Cohen $\left.-f^{2}=1.13\right)$. According to this model, teamwork abilities, loneliness, subjective well-being, age, sex (female), and nursing school (public), appeared as influencing factors in the development of empathy in patient care. Teamwork abilities, being a female student, and studying in a public nursing school, showed a positive linear relationship with empathy. On the contrary, loneliness, subjective well-being and age, showed a negative influence in the variability of empathy. A summary of this analysis is shown in Table 4.

This model complied with all the conditions necessary for statistic inference: assumptions of normality of residuals, homogeneity of residuals variance, linearity, no

Table 1 Descriptive Statistics and Cronbach's alpha coefficients

\begin{tabular}{|c|c|c|c|c|c|}
\hline & JSE-HPS $^{\mathrm{a}}$ & JSAPNC $^{\mathbf{b}}$ & JeffSPLL-HPS ${ }^{c}$ & SELSA-S ${ }^{d}$ & $\mathrm{SWLS}^{\mathrm{e}}$ \\
\hline$n$ & 687 & 690 & 690 & 685 & 696 \\
\hline \multicolumn{6}{|l|}{ Global scores } \\
\hline Possible Range & $20-140$ & $15-60$ & $14-56$ & $15-105$ & $5-25$ \\
\hline Actual Range & $38-138$ & $15-60$ & $15-56$ & $15-96$ & $5-25$ \\
\hline Mean (M) & 102.02 & 49.98 & 43.93 & 47.18 & 18.05 \\
\hline Median (Mdn) & 104 & 52 & 45 & 47 & 19 \\
\hline Interquartile range (IQR) & 25 & 9 & 8 & 22 & 7 \\
\hline Standard Deviation (SD) & 17.85 & 8.90 & 7.64 & 15.24 & 5.80 \\
\hline Cronbach's alpha & 0.84 & 0.91 & 0.88 & 0.77 & 0.82 \\
\hline
\end{tabular}

a Jefferson Scale of Empathy

b Jefferson Scale of Attitudes toward Physician-Nurse Collaboration

c Jefferson Scale of Lifelong Learning

${ }^{d}$ Social and Emotional Loneliness Scale for Adults

e Satisfaction with Life Scale 
Table 2 Two-Way ANOVA for empathy in undergraduate nursing students $(n=700)$

\begin{tabular}{lrrrr}
\hline Source of variation & $\left.\boldsymbol{F}_{1,680}\right)$ & $\boldsymbol{\eta}^{\mathbf{2}}$ & $\boldsymbol{\eta}_{\mathbf{p}}{ }^{\mathbf{2}}$ & $\boldsymbol{p}$ \\
\hline Main Effects & & & & \\
Sex (men vs. women) & 9.51 & 0.01 & 0.01 & 0.002 \\
Nursing school (public vs. private) & 25.82 & 0.04 & 0.04 & $<0.001$ \\
$\begin{array}{l}\text { Two-Way Interaction } \\
\text { Sex-Nursing school }\end{array}$ & 15.04 & 0.02 & 0.02 & $<0.001$ \\
\hline
\end{tabular}

$F$, F value; $\eta^{2}$, Eta-squared; $\eta_{p}{ }^{2}$, Eta-partial-square; $p$, $p$-Value

auto-correlation and no multicollinearity. In addition, separate linear regression analyses were performed using teamwork, loneliness, lifelong learning, and life satisfaction (subjective well-being) as dependent variables while all the others were used as potential predictors. These analyses were performed in order to explore whether these variables were sensitive to the influence of empathy and other variables collected. However, none of the models obtained fulfilled all conditions for statistical inference.

Finally, with the purpose of having a better understanding of the subjective well-being's role, this variable

Table 3 Spearman's coefficients between empathy (JSE-HPS) and variables with possible role of influence

\begin{tabular}{|c|c|c|}
\hline Variables & $\rho$ & $p$ \\
\hline \multicolumn{3}{|l|}{ Professionalism competencies } \\
\hline Teamwork (JSAPNCª) & +0.59 & $<0.001$ \\
\hline Lifelong learning (JeffSPLL-HPS') & +0.39 & $<0.001$ \\
\hline \multicolumn{3}{|l|}{ Subjective well-being } \\
\hline Life satisfaction $\left(S W L S^{\complement}\right.$ ) & +0.01 & 0.72 \\
\hline \multicolumn{3}{|l|}{ Relationship with parents } \\
\hline Positive mother's relationship & +0.08 & 0.03 \\
\hline Positive father's relationship & +0.06 & 0.13 \\
\hline \multicolumn{3}{|l|}{ Nursing studies } \\
\hline Semester of enrolment & +0.06 & 0.14 \\
\hline \multicolumn{3}{|l|}{ Loneliness (SELSA-S ${ }^{\mathrm{d}}$ ) } \\
\hline Global measure & -0.41 & $<0.001$ \\
\hline Romantic dimension & -0.02 & 0.67 \\
\hline Social dimension & -0.43 & $<0.001$ \\
\hline Family dimension & -0.45 & $<0.001$ \\
\hline Age & -0.15 & $<0.001$ \\
\hline
\end{tabular}

Table 4 A multiple regression model for global scores of the JSE-HPS in nursing students

\begin{tabular}{lllll}
\hline Predictors & $\boldsymbol{\beta}$ & $\boldsymbol{S E}$ & $\boldsymbol{t}$ & $\boldsymbol{p}$ \\
\hline Teamwork (JSAPNC ${ }^{\mathrm{a}}$ ) & +1.16 & 0.06 & +18.81 & $<0.001$ \\
Loneliness (SELSA-S ${ }^{\mathrm{b}}$ ) & -0.27 & & -6.90 & \\
Satisfaction with life (SWLS $)$ & -0.31 & & -2.64 & \\
Age & -0.19 & & -2.23 & 0.001 \\
Sex [women] & & 0.09 & & 0.03 \\
Nursing school [public] & +4.27 & 1.56 & +2.73 & 0.007 \\
\hline B, & +3.52 & 1.11 & +3.19 & 0.002 \\
\hline
\end{tabular}

$\beta$, beta coefficient; $S E$, standard error; $t, t$-experimental; $p, p$-value

a Jefferson Scale of Attitudes toward Physician-Nurse Collaboration

${ }^{\mathrm{b}}$ Social and Emotional Loneliness Scale for Adults

'Satisfaction with Life Scale

was compared by university and sex. Differences appeared in the first case $(p<0.001)$, but not in the second one $(p=0.60)$, confirming a different pattern in the selfperception of personal life according to nursing school (Fig. 1).

\section{Discussion}

The JSE-HPS showed adequate psychometric properties with Cronbach's alpha coefficient higher than the international recommendation of 0.70 set by the American Educational Research Association [48]. Results observed in the present study support the use of the Spanish version of the JSE-HPS as a consistent measure of empathy in Peruvian nursing students. These findings are also similar to those previously reported in nursing students from different cultural contexts such as the United States [54, 55], Mexico [35], Italy [27], Spain [44], Peru [9, 34], or Albania [26].

Regarding the first objective, our findings confirmed differences in empathy measurements in nursing students by sex, nursing school and the interaction of both. These findings, regarding sex, are in consonance with previous studies performed in other cultural settings in

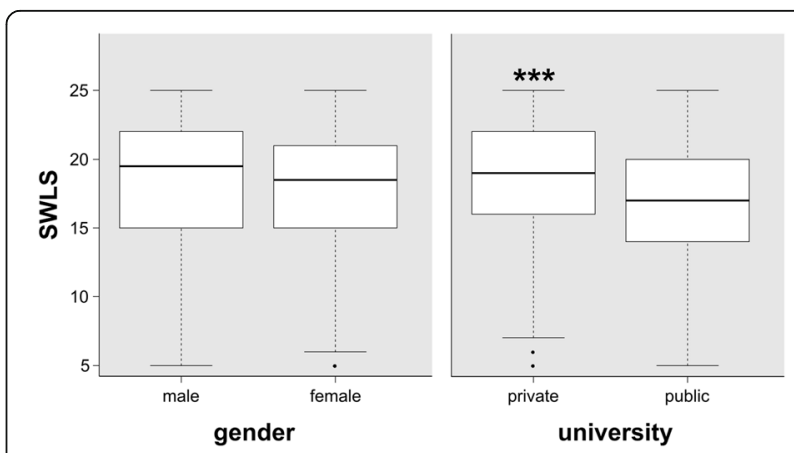

Fig. 1 Scores on Satisfaction with Life scale (SWLS) by gender and university; ${ }^{* * *} p<0.001$ 
nursing students and other non-medicine healthcare students where female students showed higher scores on empathy in comparison with their male peers $[26,27$, 55-57]. On the other hand, differences observed by nursing school (public vs. private) suggest that, in the absence of a targeted training in empathy, the social environment surrounding the university acquires an important role of influence in the development of empathy in nursing students. This finding brings evidence supporting the idea, proposed by Susanne Täuber, that social environments influence human relationships and social interactions [58]. Elitism, social stereotypes and racial bias, appear as possible manners of this influence. Elitism: In Peru, similarly to other countries, studying in a private university implies an important economic investment for students and their families, not always accessible for everyone. Elitism among students enrolled in these institutions is a social consequence derived from it. In the US, this elitism has been characterized as an influencing factor in the lack of altruist and lower scores in empathy measures in American medical students enrolled in high-ranked institutions [59, 60]. Social stereotypes and racial bias: Social influence can be also strengthening by the effect of cultural and racial stereotypes that are still dominant in the Peruvian society [61]. Indeed, studies in social psychology and neurosciences have revealed that empathic responses may be reduced by social stereotypes and racial bias [62, 63]. Findings observed from the comparison analysis of the scores of the Satisfaction with Life Scale by university groups may reinforce this interpretation.

The second objective was to determine the type of association between empathy and the other variables measured. Empathy showed a positive correlation with measures of teamwork and lifelong learning. These findings bring evidence supporting the theory that these three competences are specific elements of a common construct: professionalism [64]. Furthermore, this finding is in consonance with others reported in Mexico, where a positive association between empathy and teamwork measures was observed in students of nursing [35]. On the contrary, empathy showed an inverse correlation with loneliness measures and age. These findings, in the case of loneliness, are in consonance with others recently reported in Chilean nurses [2] and Spanish nursing students [44]. Age, but not semester of enrolment, showed an inverse association with empathy indicating that this association was due to the students' age and not by the semester in which the student was attending. Taking this into account, this association may be consequence of the social environment, previously described. In this sense, it is possible that older students become less idealistic and carry more social prejudgments than their younger peers, and this difference is reflected in a lower score in empathy.
Finally, the third objective was to characterize variables as predictors of empathy's measurements. A linear regression analysis confirmed preliminary findings indicating that teamwork, sex (being a female student) and studying in a public nursing school, are positive predictors of empathy. On the contrary, age, loneliness and subjective well-being appeared as negative predictors of empathy. It is not surprising that subjective well-being plays a negative role of influence in the development of empathy after taking into consideration the differences observed when private and public university groups were compared. In the absence of a targeted training on empathy, it is possible that students enrolled in private universities have less personal resources for empathizing with poor patients. On the contrary, students from public universities probably have more personal resources at the moment to understand and communicate with patients who have to straggling with economic issues related to their treatments.

\section{Limitations and strengths}

To appreciate the findings of this study, some limitations require consideration. First, the design of the study was a cross-sectional self-reporting questionnaire. Selfreporting could lead to response bias or socialdesirability bias. Second, analyses are based upon a convenient sample of nursing students from one Peruvian region. And third, loneliness, one of the variables measured, is described as a multiple dimensional concept that can be analysed as a global construct or as separate domains. However, inference analysis performed in this study allowed only a global characterization of loneliness since the models did not fit with all necessary conditions when using separate domains.

On the contrary, the strengths of this study are: the large sample size, which provides great statistical power to analyse the current level of knowledge and elements measured; and the good psychometric properties of the instruments, The sample was composed by nursing students (both from public and private universities) enrolled in schools without targeted educative activities focused to the improvement of empathy, offering an unique opportunity to analyse the real effect that nonacademic factors play in the early development of this ability. Another strength is related to the fact that Cusco city has a significant relevance in other cultural contexts, due to its multicultural and multilingual social structure.

\section{Implications}

Empathy is a core competence in nursing. To understand the main factors influencing empathy, which are not directly related with formal curricula, is highly important for educators. Findings observed in this study bring valuable information of non-academic elements 
that are influencing the empathic ability of nursing students. This knowledge acquires more relevance under current circumstances of the pandemic since many nursing schools are adapting their curricula to e-learning or blended methodologies. This drastic change has important implications in the training of empathic abilities that require intensive social contact. Staying at home privileges the influence that other aspects, associated to the social environments (such as family) and personal resources, have in the early development of this ability. Future research should explore nursing students' behaviour regarding communication and understanding abilities associated with empathy after the pandemic is over in order to determine suitable methodological strategies for its enhancement in safety conditions.

\section{Conclusions}

Findings have indicated that, in the absence of targeted training on empathy, this ability is sensitive to the influence of personal characteristics and of the social and family environments. Genetic predisposition associated with gender, personal life experience, social skills, and loneliness appear as important factors of influence associated with personal characteristics. On the other hand, the social environment and the family appear as two important influencing factors in the development of empathy.

\section{Abbreviations}

JSE-HPS: Healthcare student's version of the JSE; JSAPNC: Jefferson Scale of Attitudes toward Physician-Nurse Collaboration; JeffSPLL-HPS: Healthcare student's version of the JeffSPLL; SELSA-S: Short version of the Social and Emotional Loneliness Scale for Adults; SWLS: Satisfaction with Life Scale

\section{Supplementary information}

The online version contains supplementary material available at https://doi. org/10.1186/s12912-021-00773-2.

\section{Additional file 1}

\section{Acknowledgements}

Authors would like to acknowledge the contribution of the Universidad Nacional San Antonio Abad del Cusco, Universidad Andina del Cusco, and Universidad Tecnologica de los Andes, all in Cusco, for their institutional support in the development of this study. An special acknowledgment to the following persons for their valuable collaboration and support along this study: Begonia Choquenaira, Luis A. Chihuantito, Sdenka Caballero, and Edo Gallegos. Finally, authors would like to acknowledge all nursing students who voluntarily participated in this study.

\section{Authors' contributions}

LV was in charge of the study's overall design and drafting of the manuscript. MSM and LV performed the statistical processing of data. NBT was in charge of the coordination with the participating institutions. PM was in charge of survey design, distribution and data collection. LV and RDB prepared the manuscript drafts. All authors contributed to the presented work, participated during the interpretation process of the results and approved the final manuscript.
Authors' information (optional)

NBT and PM have contributed equally in this work and should be considered as the joint first authors.

\section{Funding}

This study was supported in Peru by the National Council of Science, Technology and Technological Innovation (CONCYTEC) and the National University San Antonio Abad del Cusco (UNSAAC), Ref. No E041-2017UNSAAC-02; and in Spain by the Institute of Health Carlos III (ISCIII), Ref. No PI16/01934, and the Operational Program of the European Regional Development Fund (FEDER-LARIOJA), Ref. 6FRS-ABC-012.

\section{Availability of data and materials}

The data generated and analysed during this study are included in this published article as a supplementary file (dataset.xlsx).

\section{Declarations}

Ethics approval and consent to participate

Ethical approval was sought and received from an independent ethical committee (Comité Ético de Investigación de La Rioja; Ref. CEICLAR-PI-199). This research was carried out in accordance with the principles of the Helsinki Declaration. All participant institutions provided administrative approval and support to the process of distribution and collection of data. Participants were informed of the purpose, content, benefits, risks, and voluntariness in participating in this study. Participants provided their written inform consent.

\section{Consent for publication}

No applicable.

\section{Competing interests}

The authors declare no potential conflicts of interest with respect to the research, authorship, and/or publication of this article.

\section{Author details}

${ }^{1}$ Universidad Nacional San Antonio Abad del Cusco, Av. de La Cultura 773, 08000 Cusco, Peru. ${ }^{2}$ Universidad de Granada, C/ Santander 1, 52005 Melilla, Spain. ${ }^{3}$ Hospital Universitario San Pedro, C/Piqueras 98, 26006 Logroño, Spain. ${ }^{4}$ Centro Nacional de Documentación en Bioética, C/Piqueras 98, 26006 Logroño, Spain. ${ }^{5}$ Centro de Investigación Biomédica de La Rioja (CIBIR), C/ Piqueras 98, La Rioja 26006 Logroño, Spain.

Received: 9 March 2021 Accepted: 29 November 2021 Published online: 08 December 2021

\section{References}

1. Vivanco L, Delgado-Bolton R. Professionalism. In: Encyclopedia of Global Bioethics. Cham: Springer International Publishing; 2015. p. 1-9. doi:https:// doi.org/10.1007/978-3-319-05544-2_353-1.

2. Marilaf Caro M, San-Martín M, Delgado-Bolton R, Vivanco L. Empathy, loneliness, burnout, and life satisfaction in Chilean nurses of palliative care and homecare services | Empatía, soledad, desgaste y satisfacción personal en Enfermeras de cuidados paliativos y atención domiciliaria de Chile. Enfermería Clínica. 2017;27:379-86. doi:https://doi.org/10.1016/j.enfcli.2017. 04.007.

3. Williams J, Stickley T. Empathy and nurse education. Nurse Educ Today. 2010;30:752-5. doi:https://doi.org/10.1016/j.nedt.2010.01.018.

4. Richardson C, Percy M, Hughes J. Nursing therapeutics: Teaching student nurses care, compassion and empathy. Nurse Educ Today. 2015;35:e1-5.

5. San-Martín M, Delgado-Bolton R, Vivanco L. Role of a semiotics-based curriculum in empathy enhancement: A longitudinal study in three dominican medical schools. Front Psychol. 2017:8:1-8.

6. Fernández-Rodríguez $L J$, Bardales-Zuta VH, San-Martín M, Delgado Bolton RC, Vivanco L. Empathy Enhancement Based on a Semiotics Training Program: A Longitudinal Study in Peruvian Medical Students. Front Psychol. 2020;11:1-10

7. Bas-Sarmiento $P$, Fernández-Gutiérrez $M$, Baena-Baños $M$, Romero-Sánchez JM. Efficacy of empathy training in nursing students: A quasi-experimental study. Nurse Educ Today. 2017:59:59-65. doi:https://doi.org/10.1016/j.nedt.2 017.08.012. 
8. Yang C, Zhu Y-L, Xia B-Y, Li Y-W, Zhang J. The effect of structured empathy education on empathy competency of undergraduate nursing interns: A quasi-experimental study. Nurse Educ Today. 2020;85:104296. doi:https://doi. org/10.1016/.jnedt.2019.104296.

9. López-Morales H, Rivera-Diaz E, Ore-Zuñiga A, Vera-Portilla A, San-Martín M, Delgado Bolton RC, et al. Positive Impact of Professionalism on the Perception of Global Well-Being: A Study in Healthcare Professionals Starting Their First Working Experience in Peruvian Rural Areas. Front Public Heal. 2020:8:1-9

10. San-Martín M, Rivera EM, Alcorta-Garza A, Vivanco L. Moral perception, educational environment, and development of medical professionalism in medical students during the clinical rotations in Peru. Int J Ethics Educ. 2016;1:163-72. doi:https://doi.org/10.1007/s40889-016-0017-8.

11. Halpern J. What is clinical empathy? J Gen Intern Med. 2003;18:670-4. doi: https://doi.org/10.1046/j.1525-1497.2003.21017.x.

12. Fernandez AV, Zahavi D. Basic empathy: Developing the concept of empathy from the ground up. Int J Nurs Stud. 2020;1 10:103695. doi:https:// doi.org/10.1016/j.jinurstu.2020.103695.

13. Hojat M. Empathy in health professions education and patient care. 2016.

14. Starcevic V, Piontek CM. Empathic understanding revisited: conceptualization, controversies, and limitations. Am J Psychother. 1997;51:317-28. doi:https://doi.org/10.1176/appi.psychotherapy.1997. 51.3.317.

15. Decety J, Jackson PL. A Social-Neuroscience Perspective on Empathy. Curr Dir Psychol Sci. 2006;15:54-8. doi:https://doi.org/10.1111/j.0963-7214.2006. 00406.x.

16. Decety J, Smith KE, Norman GJ, Halpern J. A social neuroscience perspective on clinical empathy. World Psychiatry. 2014;13:233-7. doi:https://doi.org/10.1 002/wps.20146.

17. Wilson NA, Ahmed RM, Hodges JR, Piguet O, Irish M. Constructing the social world: Impaired capacity for social simulation in dementia. Cognition. 2020; 202:104321. doi:https://doi.org/10.1016/j.cognition.2020.104321.

18. Hassabis D, Kumaran D, Vann SD, Maguire EA. Patients with hippocampal amnesia cannot imagine new experiences. Proc Natl Acad Sci U S A. 2007; 104:1726-31.

19. Dean $S$, Halpern J, McAllister M, Lazenby M. Nursing education, virtual reality and empathy? Nurs open. 2020;7:2056-9. doi:https://doi.org/10.1002/ nop2.551.

20. Smith KE, Norman GJ, Decety J. The complexity of empathy during medical school training: evidence for positive changes. Med Educ. 2017:51:1146-59.

21. Cheng Y, Lin CP, Liu HL, Hsu YY, Lim KE, Hung D, et al. Expertise Modulates the Perception of Pain in Others. Curr Biol. 2007;17:1708-13.

22. Kogler L, Müller VI, Werminghausen E, Eickhoff SB, Derntl B. Do I feel or do I know? Neuroimaging meta-analyses on the multiple facets of empathy. Cortex. 2020;129:341-55. doi:https://doi.org/10.1016/j.cortex.202 0.04.031.

23. Fernandez AV, Zahavi D. Can we train basic empathy? A phenomenological proposal. Nurse Educ Today. 2020;98:104720. doi:https://doi.org/10.1016/j. nedt.2020.104720.

24. Hojat M, DeSantis J, Shannon SC, Speicher MR, Bragan L, Calabrese LH. Empathy as related to gender, age, race and ethnicity, academic background and career interest: A nationwide study of osteopathic medical students in the United States. Med Educ. 2020;54:571-81. doi:https://doi. org/10.1111/medu.14138

25. Guilera T, Batalla I, Forné C, Soler-González J. Empathy and big five personality model in medical students and its relationship to gender and specialty preference: a cross-sectional study. BMC Med Educ. 2019;19:57. doi: https://doi.org/10.1186/s12909-019-1485-2.

26. Petrucci C, Gaxhja E, La Cerra C, Caponnetto V, Masotta V, Dante A, et al. Empathy Levels in Albanian Health Professional Students: An Explorative Analysis Using the Jefferson Scale of Empathy. SAGE Open. 2021;11: 215824402110321. doi:https://doi.org/10.1177/21582440211032192.

27. Montanari P, Petrucci C, Russo S, Murray I, Dimonte V, Lancia L. Psychometric properties of the Jefferson Scale of Empathy-Health Professional Student's version: An Italian validation study with nursing students. Nurs Heal Sci. 2015;17:483-91.

28. Christov-Moore L, Simpson EA, Grigaityte K, lacoboni M, Ferrari PF, Sciences B, et al. Empathy: Gender effects in brain and behaviour. 2016.

29. Hojat M, D P, Gonnella JS, Nasca TJ, Mangione S, Vergare M, et al. Measurement, and Relationship to Gender and Specialty. Psychiatry Interpers Biol Process. 2002;159:1563-9.
30. Costa P, Alves R, Neto I, Marvão P, Portela M, Costa MJ. Associations between medical student empathy and personality: A multi-institutional study. PLoS One. 2014;9:1-7.

31. Piumatti G, Abbiati M, Baroffio A, Gerbase MW. Empathy trajectories throughout medical school: relationships with personality and motives for studying medicine. Adv Health Sci Educ Theory Pract. 2020;25:1227-42.

32. Bach RA, Defever AM, Chopik WJ, Konrath SH. Geographic variation in empathy: A state-level analysis. J Res Pers. 2017;68:124-30. doi:https://doi. org/10.1016/j.jp.2016.12.007.

33. Alcorta-Garza A, San-Martín M, Delgado-Bolton R, Soler-González J, Roig H, Vivanco L. Cross-Validation of the Spanish HP-Version of the Jefferson Scale of Empathy Confirmed with Some Cross-Cultural Differences. Front Psychol. 2016;7:1002. doi:https://doi.org/10.3389/fpsyg.2016.01002.

34. Berduzco-Torres N, Choquenaira-Callañaupa B, Medina P, Chihuantito-Abal LA, Caballero S, Gallegos E, et al. Factors Related to the Differential Development of Inter-Professional Collaboration Abilities in Medicine and Nursing Students. Front Psychol. 2020;11:432.

35. Tuirán-Gutiérrez GJ, San-Martín M, Delgado-Bolton R, Bartolomé B, Vivanco L. Improvement of Inter-Professional Collaborative Work Abilities in Mexican Medical and Nursing Students: A Longitudinal Study. Front Psychol. 2019;10: 5. doi:https://doi.org/10.3389/fpsyg.2019.00005.

36. Ponnamperuma G, Yeo SP, Samarasekera DD. Is empathy change in medical school geo-socioculturally influenced? Med Educ. 2019;53:655-65.

37. Hojat M, Zuckerman M, Magee M, Mangione S, Nasca T, Vergare M, et al. Empathy in medical students as related to specialty interest, personality, and perceptions of mother and father. Pers Individ Dif. 2005;39:1205-15. doi:https://doi.org/10.1016/j.paid.2005.04.007.

38. Berduzco-Torres N, Medina P, Choquenaira-Callañaupa B, San-Martín M, Delgado Bolton RC, Vivanco L. Family Loneliness: Its Effects in the Development of Empathy, Teamwork and Lifelong Learning Abilities in Medical Students. Front Psychol. 2020;11:1-10.

39. Hojat M, Fields SK, Veloski JJ, Griffiths M, Cohen MJM, Plumb JD. Psychometric Properties of an Attitude Scale Measuring Physician-Nurse Collaboration. Eval Health Prof. 1999;22:208-20. doi:https://doi.org/10.1177/ 01632789922034275.

40. Kosht Novak M, Palladino C, Ange B, Richardson D, Novak MK, Palladino C et al. Measuring health professions students' orientation toward lifelong learning. J Allied Health. 2014;43:146-9.

41. Hojat M, Veloski JJ, Gonnella JS. Measurement and correlates of physicians' lifelong learning. Acad Med. 2009;84:1066-74. doi:https://doi.org/10.1097/A CM.0b013e3181acf25f.

42. DiTommaso E, Brannen C, Best LA. Measurement and Validity Characteristics of the Short Version of the Social and Emotional Loneliness Scale for Adults. Educ Psychol Meas. 2004;64:99-1 19. doi:https://doi.org/10.1177/0013164403258450.

43. Soler-Gonzalez J, San-Martín M, Delgado-Bolton R, Vivanco L. Human connections and their roles in the occupational well-being of healthcare professionals: A study on loneliness and empathy. Front Psychol. 2017:8:1475.

44. Dominguez V, San-Martín M, Vivanco L, Domínguez V, San-Martín M, Vivanco L. Relaciones familiares, soledad y empatía en el cuidado del paciente en estudiantes de enfermería. Aten Primaria. 2017;49:6-12. doi: https://doi.org/10.1016/..aprim.2016.03.007.

45. Atienza FL, Pons D, Balaguer I, García-Merita M. Propiedades Psicométricas de la Escala de Satisfaccióncon la Vida en Adolescentes. Psicothema. 2000; 12:314-9.

46. Diener E, Emmons RA, Larsen RJ, Griffin S. The satisfaction with Life Scale. J Pers Assess. 1985;49:71-5. doi:https://doi.org/10.1207/s15327752jpa4901_13.

47. Vanderweele TJ, Hawkley LC, Cacioppo JT. On the reciprocal association between loneliness and subjective well-being. Am J Epidemiol. 2012;176:777-84.

48. AERA, APA, NCME. Estándares para pruebas educativas y psicológicas (M. Lieve, Trans.). Washington, DC: American Educational Research Association; 2018.

49. Cohen J. Statistical Power Analysis for the Behavioral Sciences. Second edi. New York: Lawrence Erlbaum Associates Publishers; 1988.

50. Navarro D. Isr: Companion to "Learning Statistics with R." The Comprehensive R Archive Network. 2015. https://cran.r-project.org/web/pa ckages//sr/. Accessed 18 Nov 2021.

51. Bliese P. multilevel: multilevel Functions. The Comprehensive R Archive Network. 2016. https:/cran.r-project.org/web/packages/multilevel/index. html. Accessed 18 Nov 2021.

52. Stanley D. apaTables: Create American Psychological Association (APA) Style Tables. The Comprehensive R Archive Network. 2021. https://cran.r-project. org/web/packages/apaTables/index.html. Accessed 18 Nov 2021. 
53. Gross J. nortest: Tests for Normality. The Comprehensive R Archive Network. 2015. https://cran.r-project.org/web/packages/nortest/index.html. Accessed 18 Nov 2021.

54. Ward J, Cody J, Schaal M, Hojat M. The Empathy Enigma: An Empirical Study of Decline in Empathy Among Undergraduate Nursing Students. J Prof Nurs. 2012;28:34-40.

55. Ward J, Schaal M, Sullivan J, Bowen ME, Erdmann JB, Hojat M. Reliability and validity of the Jefferson Scale of empathy in undergraduate nursing students. J Nurs Meas. 2009;17:73-88.

56. Williams B, Brown T, McKenna L, Boyle MJ, Palermo C, Nestel D, et al. Empathy levels among health professional students: a cross-sectional study at two universities in Australia. Adv Med Educ Pract. 2014;5:107-13. doi: https://doi.org/10.2147/AMEP.S57569.

57. Wilson SE, Prescott J, Becket G. Empathy levels in first- and third-year students in health and non-health disciplines. Am J Pharm Educ. 2012;76:24. doi:https://doi.org/10.5688/ajpe76224.

58. Täuber S. Moralized Health-Related Persuasion Undermines Social Cohesion. Front Psychol. 2018;9:1-14. doi:https://doi.org/10.3389/fpsyg.2018.00909.

59. van Ryn M, Hardeman RR, Phelan SM, Burke SE, Przedworski J, Allen ML, et al. Psychosocial predictors of attitudes toward physician empathy in clinical encounters among 4732 1st year medical students: A report from the CHANGES study. Patient Educ Couns. 2014;96:367-75. doi:https://doi. org/10.1016/j.pec.2014.06.009.

60. Li J, Dow WH, Kariv S. Social preferences of future physicians. Proc Natl Acad Sci. 2017;114:E10291-300. doi:https://doi.org/10.1073/pnas.1705451114.

61. Genna K, Espinosa A. Identidad, etnicidad y bienestar social en un contexto socialmente excluyente. Psicol Soc. 2012;24:84-93. doi:https://doi.org/10.1 590/S0102-71822012000100010

62. Azevedo RT, Macaluso E, Avenanti A, Santangelo V, Cazzato V, Aglioti SM. Their pain is not our pain: brain and autonomic correlates of empathic resonance with the pain of same and different race individuals. Hum Brain Mapp. 2013;34:3168-81. doi:https://doi.org/10.1002/hbm.22133.

63. Avenanti A, Sirigu A, Aglioti SM. Racial bias reduces empathic sensorimoto resonance with other-race pain. Curr Biol. 2010;20:1018-22. doi:https://doi. org/10.1016/j.cub.2010.03.071

64. Veloski JJ, Hojat M. Measuring specific elements of Professionalism: Empathy, Teamwork, and Lifelong learning. In: Stern DT, editor. Measuring medical professionalism. New York: Oxford University Press; 2006. p. 117-46.

\section{Publisher's Note}

Springer Nature remains neutral with regard to jurisdictional claims in published maps and institutional affiliations.

Ready to submit your research? Choose BMC and benefit from:

- fast, convenient online submission

- thorough peer review by experienced researchers in your field

- rapid publication on acceptance

- support for research data, including large and complex data types

- gold Open Access which fosters wider collaboration and increased citations

- maximum visibility for your research: over $100 \mathrm{M}$ website views per year

At $\mathrm{BMC}$, research is always in progress.

Learn more biomedcentral.com/submissions 